\title{
Patient-reported measurement of time to diagnosis in cancer: development of the Cancer Symptom Interval Measure (C-SIM) and randomised controlled trial of method of delivery
}

Richard D Neal ${ }^{1 *}$, Sadia Nafees ${ }^{1}$, Diana Pasterfield ${ }^{1}$, Kerenza Hood ${ }^{2}$, Maggie Hendry ${ }^{1}$, Simon Gollins ${ }^{3}$, Matthew Makin ${ }^{3}$, Nick Stuart ${ }^{3}$, Jim Turner ${ }^{3}$, Ben Carter ${ }^{2}$, Clare Wilkinson ${ }^{1}$, Nefyn Williams ${ }^{1}$ and Mike Robling ${ }^{2}$

\begin{abstract}
Background: The duration between first symptom and a cancer diagnosis is important because, if shortened, may lead to earlier stage diagnosis and improved cancer outcomes. We have previously developed a tool to measure this duration in newly-diagnosed patients. In this two-phase study, we aimed further improve our tool and to conduct a trial comparing levels of anxiety between two modes of delivery: self-completed versus researcher-administered.
\end{abstract}

Methods: In phase 1, ten patients completed the modified tool and participated in cognitive debrief interviews. In phase 2, we undertook a Randomised Controlled Trial (RCT) of the revised tool (Cancer Symptom Interval Measure $(C-S I M))$ in three hospitals for 11 different cancers. Respondents were invited to provide either exact or estimated dates of first noticing symptoms and presenting them to primary care. The primary outcome was anxiety related to delivery mode, with completeness of recording as a secondary outcome. Dates from a subset of patients were compared with GP records.

Results: After analysis of phase 1 interviews, the wording and format were improved. In phase 2, 201 patients were randomised (93 self-complete and 108 researcher-complete). Anxiety scores were significantly lower in the researcher-completed group, with a mean rank of 83.5; compared with the self-completed group, with a mean rank of 104.0 (Mann-Whitney $U=3152, p=0.007$ ). Completeness of data was significantly better in the researcher-completed group, with no statistically significant difference in time taken to complete the tool between the two groups. When comparing the dates in the patient questionnaires with those in the GP records, there was evidence in the records of a consultation on the same date or within a proscribed time window for 32/37 (86\%) consultations; for estimated dates there was evidence for 23/37 consultations (62\%).

Conclusions: We have developed and tested a tool for collecting patient-reported data relating to appraisal intervals, help-seeking intervals, and diagnostic intervals in the cancer diagnostic pathway for 11 separate cancers, and provided evidence of its acceptability, feasibility and validity. This is a useful tool to use in descriptive and epidemiological studies of cancer diagnostic journeys, and causes less anxiety if administered by a researcher.

Trial registration: ISRCTN04475865

Keywords: Cancer symptoms, Patient intervals, Appraisal, Primary care intervals, Diagnosis, Randomised controlled trial, Tool development

\footnotetext{
* Correspondence: r.neal@bangor.ac.uk

${ }^{1}$ North Wales Centre for Primary Care Research, Bangor University, Gwenfro

Unit 5, Wrexham Technology Park, Wrexham LL13 7YP, UK

Full list of author information is available at the end of the article
} 


\section{Background}

Mortality from cancer is worse in the UK than most other European countries [1,2]. The reasons for this are multi-factorial, but diagnostic delays and consequent later stage diagnoses are likely to be major contributory factors [3]. Interventions to reduce diagnostic delays, which result in a less advanced stage at diagnosis, have the potential to improve cancer survival [4], although tumour biology is also important. Interventions need to account for lead-time bias whereby more timely diagnosis may improve survival by bringing forward the diagnosis rather than delaying mortality. Diagnostic delays (perhaps better referred to as 'time-intervals' since there is not always a 'delay') may occur throughout the cancer diagnostic pathway. Whilst a minority of patients are diagnosed through screening (in some cancers), and some present as an emergency to A\&E or via inter-specialty referral (without consulting in primary care), the majority of diagnoses are made for patients who follow the 'typical' cancer journey involving symptomatic presentation through primary care [5-8]. In the UK, and elsewhere, there has been a drive in policy towards early, and more timely diagnosis of cancer; for example the National Awareness and Early Diagnosis Initiative (England), the Detect Cancer Early Initiative (Scotland) and the International Cancer Benchmarking Partnership (several countries).

The duration between first symptom and cancer diagnosis is important because, if shortened, it may lead to earlier stage diagnosis and improved cancer outcomes $[9,10]$. Measurement is complex because some symptoms are simply present or absent (e.g. a breast lump or rectal bleeding), whilst others are not instantly noticeable (e.g. tiredness or weight loss). Most studies reporting both 'appraisal intervals' ('time taken to interpret bodily changes/symptoms') and 'help seeking' intervals (time taken to act on those interpretations and seek help') [11] use tools that ignore existing models of patient behaviour [12,13], are poorly or inadequately validated, and are open to bias. There is a well-recognised need to develop valid instruments for measuring 'delay' [11], and this is one of the recommendations of the Aarhus checklist on the design and reporting of early cancer diagnosis studies that has recently been published [14].

We previously reported the first phase of the pilot work to develop and pilot a postal version of such a tool (the 'DELAYS' tool) [15]. This questionnaire was tailored to individual cancers and asked patients to recall the dates of the onset of symptoms (based on referral guidance symptoms) and their presentation to primary care, in addition to socio-demographic and health questions. One issue that arose (predominantly from phone calls from potential respondents to the research team) was the potential anxiety that may be generated by use of the tool (for example asking patients to recall when they first experienced symptoms may cause upset if they feel that their diagnosis was unduly delayed). The other main issue was that the response rate to the postal questionnaire was only moderate (46.2\%).

Hence, the aim of this paper is to report the further development of the 'DELAYS' tool, now renamed the Cancer Symptom Interval Measure (C-SIM), through indepth cognitive testing, and its testing in a randomised controlled trial (RCT) comparing different methods of delivery (on the premise that anxiety may be less in the presence of a researcher). The primary objective of this RCT was to compare the level of patients' anxiety between two methods of delivery (self-completed and researchercompleted) of administering a tool, which measures time from first symptom to cancer diagnosis. Secondary objectives of this trial were: to compare the difference in completion rates between two methods of delivery; to describe the process, and difficulties associated with either method of delivery; to compare the information about symptoms from the patient tool with that obtained from primary care records. Hence, we gathered further information in the trial about the quality of the tool, primarily acceptability (does it provoke undue anxiety; completion rates, completion time) and feasibility (problems with administration). Focused qualitative evaluation presented in the paper (cognitive interviews) adds to the evidence for the measure's face validity, and the comparison of selfreport against records adds to the measure's criterion validity $[16,17]$.

\section{Methods}

Phase 1. Further development of the tool and cognitive debriefing interviews with cancer patients.

The aim of Phase 1 was to improve the previously reported DELAYS tool [15], to make it suitable for use in the trial, building on the previous work and making it more acceptable, more likely to be completed, and less likely to cause anxiety. This further development was informed by the cognitive aspects of survey methodology approach [18-20] and utilised the findings from systematic review evidence regarding survey response [21-23]. We recruited ten patients to complete the modified tool and participate in cognitive debriefing interviews. They were either allocated to self-completed or researchercompleted mode. In addition to asking patients questions about symptoms related to their own cancer, we also asked about the wording of questions relating to symptoms of other cancers. Post completion, the participants underwent a cognitive debriefing interview. Areas identified as problematic in the initial piloting [15] were specifically addressed.

Key interview probes were used that directly and intentionally match the steps of the standard cognitive model for survey response (comprehension, retrieval, 
judgement, response formulation) [19]. These probes were:

- What the respondent believed the question to be asking.

- What specific words and phrases meant to the respondent.

- The type of information the respondent needed to recall in order to answer the question.

- What types of strategy the respondent used to retrieve information.

- Whether the respondent devoted sufficient mental effort to answer accurately and thoughtfully.

- Whether the respondent wanted to tell the truth, or whether the respondent wanted to say something that made him/her look better (i.e. social desirability).

- Whether the respondent matched his or her internally generated answer to the response categories given by the survey question.

The data were analysed to identify dominant trends across interviews and to identify 'discoveries' (unexpected problems that were only present in one interview, but were important). Findings from these interviews informed further re-wording of the tool. Lastly, we worked with three patient groups within the North East Wales NHS Trust (now part of Betsi Cadwaladr University Health Board) to ensure that the tool captured the diagnostic journey in a way that was acceptable and easy to understand.

\section{The final tool}

This revised tool (C-SIM), in 11 separate versions (lung, colorectal, breast, pancreatic, gastric/oesophageal, renal/ bladder, endometrial/cervical, haematological, ovarian, prostate and testicular cancers), was then tested in the RCT. It comprised questions concerning the timing of the onset and presentation of symptoms. Respondents were invited to provide either exact or estimated dates of first noticing symptoms and presenting them to primary care. These covered cancer site specific symptom questions (variable between cancers); and four general cancer symptom questions (loss of appetite, weight loss, fatigue and 'feeling different'). One question asked whether they had been sent for any GP-initiated tests. Further questions covered employment status, educational qualifications, ethnicity, whether they lived alone, comorbidity, smoking status, family risk of cancer. Finally, as part of the RCT a measure of anxiety, the State-Trait Anxiety Inventory (STAI) was added as the main outcome measure [24]. The tool was produced on double sided A4 paper and was 11 pages long (see Additional file 1-the colorectal tool, and Additional file 2-the cancer specific questions for the other 10 versions). The final version of the tool built upon that previously reported [15] in that we:

- Removed the calendar landmarking tool, because participants reported that they didn't find it useful

- Added a personal letter from the Principal. Investigator inside the front cover, to try to improve the response rate [22].

- Improved the format of the boxes for the questions regarding symptom duration.

- Added more tests (CT scan \& ultrasound scan), that were missing from the original.

- Made multiple changes to the wording to make it more patient-centred and easier to understand.

Phase 2. Randomised controlled trial of method of delivery of the tool.

\section{Setting and participants}

The trial took place in the three district general hospitals in North Wales (Wrexham Maelor Hospital, Ysbyty Gwynedd, and Ysbyty Glan Clywd), now all part of Betsi Cadwaladr University Health Board. Fieldwork was undertaken by research officers/nurses from the NISCHR Clinical Research Collaboration. The research officers/nurses attended MDT meetings for the following cancers: breast, colorectal, lung, gynaecological, urological, upper GI, and haematological. At these meetings all patients with new primary diagnoses were alerted to the researcher, who then liaised with the clinical nurse specialists and other members of the clinical teams in order to assess eligibility for inclusion. Patients were eligible if they had a new diagnosis of one of the 11 cancers, were aged 18 years or above (with no upper age limit), were neither too ill nor close to death at diagnosis, were not detected by screening (some cervix, breast and colorectal), and were mentally and linguistically competent to complete the tool.

Eligible patients were then sent a letter from the clinical team inviting participation, enclosing a patient information sheet, a return FREEPOST envelope and a contact form. The researchers then contacted patients who responded positively and arranged to meet them in order to obtain consent (for data collection and access to primary care records), randomisation and tool completion. Reminders letters were sent after two weeks.

Participants randomised to the self-completion arm were handed the final tool (see above) and asked to complete it. For those randomised to the researchercompletion arm, the researcher sat with the participant in a quiet place and went through the tool with them, reading out the questions and entering their answers. Identical tools were used for both groups. All of the researchers received training to ensure that the delivery of 
the tool was as standardised as possible. Standard prompts in response to questions for both groups were permitted.

The trial was conducted from February to December 2009. Data were entered using Cardiff TeleForm (optical character recognition system); 22 questionnaires (two for each cancer site) were manually checked.

\section{Randomisation}

Patients were randomised to either the researchercompleted or the self-completed mode. A computer generated random number sequence based on random permuted blocks of sizes 4, 6 and 8 was used. Allocation concealment was done by the controlled use of sequentially numbered opaque envelopes, stratified by cancer site and by hospital, except for: pancreatic, testicular and ovarian cancer where the expected prevalence was too small to stratify by hospital and a block size of 4 was used to minimise the risk of chance imbalance; and haematological, endometrial/cervix that were stratified by hospital but were also randomised using a block size of 4 .

\section{Outcome measures \\ Measuring anxiety}

We used the six-item State-Trait Anxiety Inventory (STAI) to determine whether respondents had anxiety related to questionnaire completion [24]. This has a low-burden, and was designed to assess short-term fluctuations in anxiety. Each item was scored 1-4, with a total score out of 24, with higher scores indicating higher anxiety.

\section{Process evaluation}

After completion of the tool, the researcher completed a brief sheet for each patient comprising the following questions (with answer options):

- How long did it take the patient to compete the questionnaire? (<5 minutes, between 5 and 10 . minutes, between 11 and 15 minutes, $>15$ minutes)

- Where did the patient complete the questionnaire? (separate room, open area, while receiving treatment).

- The researcher answered one further question for the self-completed group only: 'Did the patient ask for any help?' (Y/N). This was to capture data relating to help sought when it had not been offered. The researcher answered two further questions for the researcher-completed group only. These were a subjective report of difficulty and anxiety observed whilst the researcher was completing the tool with each patient:

- Did the patient find any of the questions difficult? $(\mathrm{Y} / \mathrm{N})$.

Was the patient made anxious? $(\mathrm{Y} / \mathrm{N})$.

\section{Data analysis}

\section{Analysis of primary outcome}

The primary outcome when comparing the method of delivery was anxiety, with the analysis carried out blind to treatment allocation. We aimed to transform the data to produce an approximately normal distribution and undertake parametric tests; if not possible then nonparametric testing was undertaken. Missing items were imputed (see findings).

\section{Analysis of secondary outcomes}

Completeness of questionnaire The difference in completeness between the two methods of delivery were compared (z statistic) on questions common to all of the respondents. Hence we analysed data for:

- The four generic cancer questions. If the respondent ticked the 'yes' box for having the particular symptom, they were then assessed as to whether the rest of the question was entered completely. If they entered dates for first noticing and first reporting (whether exact or estimated), or they reported that they didn't tell their GP or nurse, it was regarded as 'complete'. Any less than this was regarded as 'not complete'.

- The demographic questions.

Process and difficulties From the post-completion sheets, we determined the process of and difficulties associated with both methods of delivery. This was descriptive other than $X^{2}$ for comparison of tool completion between modes of delivery.

Comparison of recall of date of first presentation against GP records We sought to obtain evidence of criterion validity by comparing the dates of consultation recorded by the measure from up to 60 patients against their GP records. A protocol was developed to identify 40-50 consecutive completed questionnaires, ensuring that there were at least 10 patients from each centre and at least three from each cancer site. Patients were excluded if they had the reported symptoms for more than two years (because unlikely to represent the first presentation of cancer). With patient consent we contacted their GP, and asked them to complete a template. We validated the date of reported first presentation of each symptom, for both exact dates and estimated dates. For exact dates, we asked GPs whether there was a consultation on that particular day, or within a two-week window either side. For estimated dates, we developed a protocol for calculating a 'pseudo-exact' time-window from the estimated date (see Additional file 3), and provided the GP with this time window for searching. We also asked the date of the actual consultation according 
to the records. For simplicity, we only analysed data for one symptom per consultation (we chose the first one that the patient listed on their questionnaire).

Time intervals in the diagnostic journey Whilst the actual time intervals in the diagnostic journey are not reported in this paper, we developed, a protocol for changing reported estimated dates into pseudo-exact dates that permit the calculation of time intervals based upon the responses obtained (Additional file 4).

\section{Sample size}

The primary aim of this study was to detect a difference in anxiety between the two methods of delivery of the tool, with the completion rates as a secondary outcome. 150 patients per group (total 300), with a 95\% CI (with a comparative completion rate of $75 \%$ ) would allow us to detect a difference between groups on response rates assuming a difference of $10 \%(95 \% \mathrm{CI}+/-9 \%)$. An overall completion rate of $75 \%$ would give 225 questionnaires for analysis of anxiety. This would give a $95 \% \mathrm{CI}$ for the difference in $\log$ anxiety for the two modes of total width 0.16 ( 0.08 from the mean to the limit). This is based on a SD of log anxiety of 0.3 (based on previous studies).

\section{Results}

Consent was obtained from 201 patients, all were randomised and completed questionnaires were received from all 201 participants (93 from self-completed and 108 from the researcher-completed) (see Figure 1: CONSORT flow diagram). A further 22 patients initially expressed an interest in taking part in the trial, but were not contacted further, because we anticipated reaching our target sample size.

1 Group similarity at baseline

The comparison between the two groups at baseline is shown in Table 1 which shows that there were no important differences between them.

2 Analysis of primary outcome The STAI scores were not normally distributed and could not be transformed into a normal distribution by either $\log$, exponential or square root transformation. Consequently non-parametric analysis was undertaken, with and without imputation of individual data if the STAI score was missing (median values imputed). 18 (9\%) missing values were replaced with the median value seven; this was carried out to allow us to assess the effect of missing values. Without imputation of missing data for the researcher-completed group $(n=107)$ the mean rank was 83.5; for the self-completed group $(n=76)$ the mean rank was 104.0 (Mann-Whitney $\mathrm{U}=3152$, $\mathrm{p}=0.007$ ). The median STAI score before replacing missing values were: researcher completed group 7.0 (IQR 3); self-completed group 8 (IQR 7). With imputation of missing data for the researchercompleted group $(\mathrm{n}=108)$ the mean rank was 91.9; for the self-completed group $(\mathrm{n}=93)$ the mean rank was 111.5 (Mann-Whitney $U=4042, p=0.014$ ). Hence STAI scores were significantly higher in the

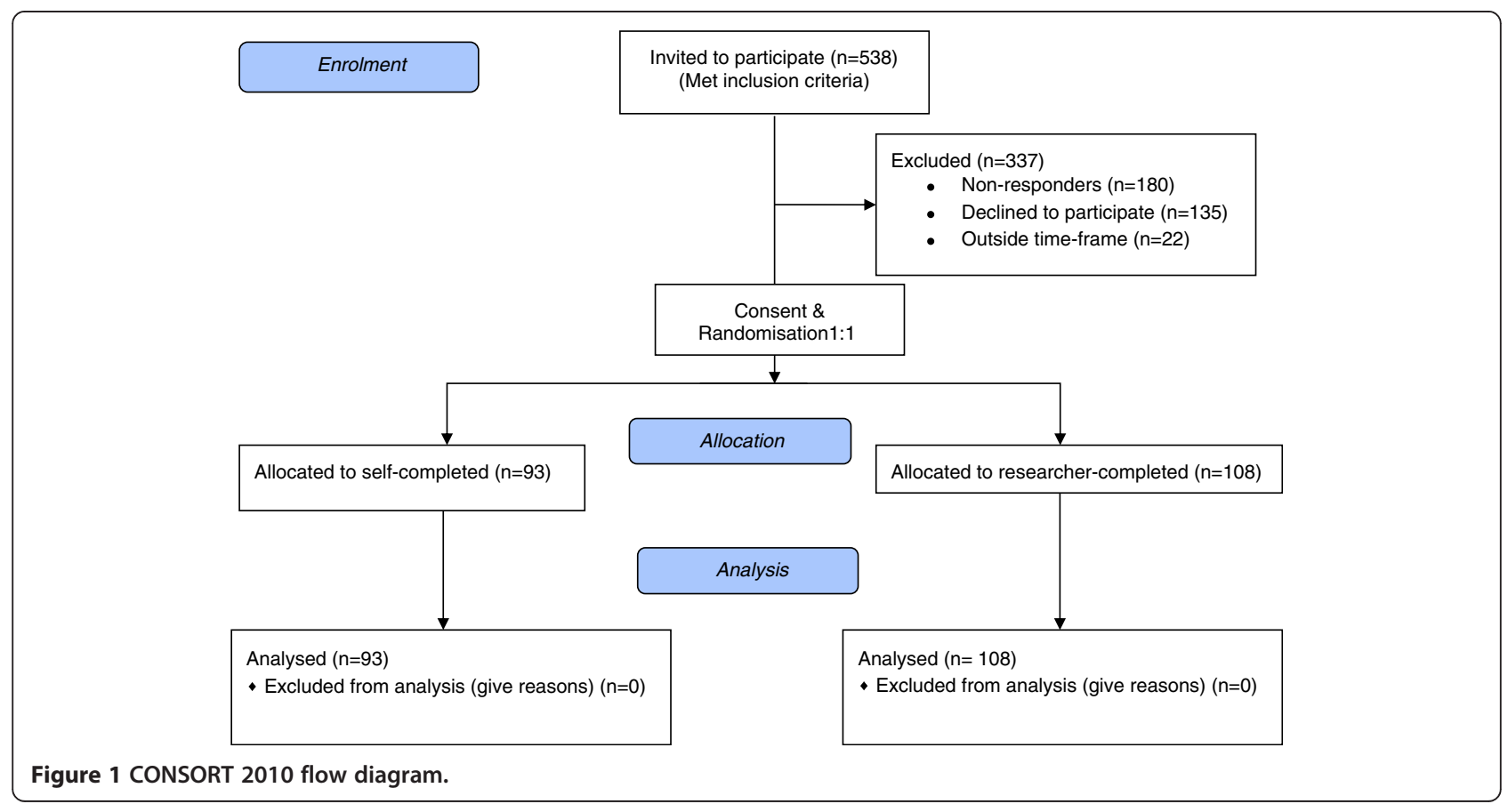


Table 1 Baseline comparison between groups

\begin{tabular}{|c|c|c|}
\hline & $\begin{array}{l}\text { Self- } \\
\text { completed } \\
n=93\end{array}$ & $\begin{array}{l}\text { Researcher- } \\
\text { completed } \\
n=108\end{array}$ \\
\hline \multicolumn{3}{|l|}{ Cancer site } \\
\hline Breast & $28(30.1 \%)$ & $28(25.9 \%)$ \\
\hline Colorectal & $15(16.1 \%)$ & $15(13.9 \%)$ \\
\hline Upper Gl & $5(5.4 \%)$ & $4(3.7 \%)$ \\
\hline Haematological & $10(10.8 \%)$ & $10(9.3 \%)$ \\
\hline Pancreas & $2(2.2 \%)$ & $3(2.8 \%)$ \\
\hline Testicle & $1(1.1 \%)$ & $1(0.9 \%)$ \\
\hline Lung & $11(11.8 \%)$ & $15(13.9 \%)$ \\
\hline Ovary & $1(1.1 \%)$ & $3(2.8 \%)$ \\
\hline Prostate & 15 16.1\%) & $21(19.4 \%)$ \\
\hline Renal/bladder & $2(2.2 \%)$ & $3(2.8 \%)$ \\
\hline Endometrium/cervix & $3(3.2 \%)$ & $5(4.6 \%)$ \\
\hline \multicolumn{3}{|l|}{ Age (years) } \\
\hline Mean (SD) & $65.3(11.3)$ & $63.6(12.2)$ \\
\hline \multicolumn{3}{|l|}{ Gender } \\
\hline Male & $45(48.4 \%)$ & $56(51.9 \%)$ \\
\hline Female & $48(51.6 \%)$ & $52(48.1 \%)$ \\
\hline \multicolumn{3}{|l|}{ Employment } \\
\hline Employed full time & $23(24.7 \%)$ & $17(15.7 \%)$ \\
\hline Employed part time & $5(5.4 \%)$ & $12(11.1 \%)$ \\
\hline Self employed full time & $6(6.5 \%)$ & $2(1.9 \%)$ \\
\hline Self employed part time & $2(2.2 \%)$ & $8(7.4 \%)$ \\
\hline Unemployed & $1(1.1 \%)$ & $2(1.9 \%)$ \\
\hline Retired & $44(47.3 \%)$ & $59(54.6 \%)$ \\
\hline Sick/disabled & $3(3.2 \%)$ & $3(2.8 \%)$ \\
\hline Looking after family/home & $4(4.3 \%)$ & $2(1.9 \%)$ \\
\hline Other & $2(2.2 \%)$ & $3(2.8 \%)$ \\
\hline Missing & $3(3.2 \%)$ & $0(0.0 \%)$ \\
\hline \multicolumn{3}{|l|}{ Educational level } \\
\hline GCSE/O Level & 19 (20.4\%) & $16(14.8 \%)$ \\
\hline A Level & $5(5.4 \%)$ & $5(4.6 \%)$ \\
\hline Diploma & $12(12.9 \%)$ & $12(11.1 \%)$ \\
\hline Degree/Higher degree & $15(16.1 \%)$ & $20(18.5 \%)$ \\
\hline Other & $11(11.8 \%)$ & $23(21.3 \%)$ \\
\hline None & $26(28.0 \%)$ & $29(26.9 \%)$ \\
\hline Missing & $5(5.4 \%)$ & $3(2.8 \%)$ \\
\hline \multicolumn{3}{|l|}{ Ethnicity } \\
\hline White British & 79 (84.9\%) & $85(78.7 \%)$ \\
\hline White & $11(11.8 \%)$ & $13(12.0 \%)$ \\
\hline White Irish & $0(0.0 \%)$ & $1(0.9 \%)$ \\
\hline Other white & $1(1.1 \%)$ & $8(7.4 \%)$ \\
\hline
\end{tabular}

Table 1 Baseline comparison between groups (Continued)

\begin{tabular}{|c|c|c|}
\hline Indian & $0(0.0 \%)$ & $1(0.9 \%)$ \\
\hline Missing & $2(2.2 \%)$ & $0(0.0 \%)$ \\
\hline \multicolumn{3}{|l|}{ Domestic status } \\
\hline Alone & $20(21.5 \%)$ & $21(19.4 \%)$ \\
\hline Spouse/partner (+/- others) & $63(67.7 \%)$ & $81(75.0 \%)$ \\
\hline Child/ren (+/- grandchildren) & $5(5.4 \%)$ & $3(2.8 \%)$ \\
\hline Sibling/s & $0(0.0 \%)$ & $1(0.9 \%)$ \\
\hline Parents & $1(1.1 \%)$ & $0(0.0 \%)$ \\
\hline Friends & $1(1.1 \%$ & $0(0.0 \%)$ \\
\hline Other & $1(1.1 \%)$ & $1(0.9 \%)$ \\
\hline Missing & $2(2.2 \%)$ & $1(0.9 \%)$ \\
\hline \multicolumn{3}{|l|}{ Co-morbidities } \\
\hline Previous cancer & $6(6.5 \%)$ & $8(7.4 \%)$ \\
\hline Diabetes & $9(9.7 \%)$ & $6(5.6 \%)$ \\
\hline COPD & $3(3.2 \%)$ & $7(6.5 \%)$ \\
\hline Asthma & $7(7.5 \%)$ & $16(14.8 \%)$ \\
\hline Other lung disease & $1(1.1 \%)$ & $2(1.9 \%)$ \\
\hline Heart disease & $11(11.8 \%)$ & $18(16.7 \%)$ \\
\hline Arthritis & $16(17.2 \%)$ & $18(16.7 \%)$ \\
\hline Peptic ulcer & $0(0.0 \%)$ & $3(2.8 \%)$ \\
\hline Irritable bowel syndrome & $8(8.6 \%)$ & $9(8.3 \%)$ \\
\hline Inflammatory bowel disease & $6(6.5 \%)$ & $5(4.6 \%)$ \\
\hline Anxiety or depression & $19(20.4 \%)$ & $23(21.3 \%)$ \\
\hline \multicolumn{3}{|l|}{ Smoking } \\
\hline Current smoker & $12(12.9 \%)$ & $16(14.8 \%)$ \\
\hline Ex-smoker & $43(46.2 \%)$ & $55(50.9 \%)$ \\
\hline Never smoked & $36(38.7 \%)$ & $37(34.3 \%)$ \\
\hline Missing & $2(2.2 \%)$ & $0(0.0 \%)$ \\
\hline \multicolumn{3}{|c|}{ At risk of cancer because of family history } \\
\hline Yes & $26(28.0 \%)$ & $23(21.3 \%)$ \\
\hline No & $65(65.9 \%)$ & $84(77.8 \%)$ \\
\hline Missing & $2(2.2 \%)$ & $1(0.9 \%)$ \\
\hline \multicolumn{3}{|c|}{ Time between diagnosis and completion (days) } \\
\hline Mean (SD) & $85.5(54.4)$ & $102.4(62.3)$ \\
\hline
\end{tabular}

self-completed group. The median STAI score after replacing missing values with the median were: researcher completed group 7.0 (IQR 3); self-completed group 7 (IQR 5.5).

3 Analysis of secondary outcome Completeness of recording The completeness of recording for the four generic cancer questions is shown in Table 2. Although numbers were small, the completeness of recording was significantly better in the researcher-completed group for one of the four generic symptoms 
Table 2 Completeness of recording for the four generic cancer symptoms

\begin{tabular}{lcccc}
\hline $\begin{array}{l}\text { Number of patients } \\
\text { reporting this symptoms }\end{array}$ & $\begin{array}{c}\text { Complete } \\
\text { data }\end{array}$ & $\begin{array}{c}\text { Incomplete } \\
\text { data }\end{array}$ & $\begin{array}{c}\mathbf{z} \\
\text { statistic }\end{array}$ & p \\
\hline Fatigue or tiredness & 53 & 2 & & \\
$\begin{array}{l}\text { Researcher-completed } \\
(n=55)\end{array}$ & 34 & 3 & -0.99 & 0.32 \\
$\begin{array}{l}\text { Self-completed }(n=37) \\
\text { Unexplained weight loss }\end{array}$ & 28 & 1 & & \\
$\begin{array}{l}\text { Researcher-completed } \\
(n=29)\end{array}$ & 23 & 1 & -0.14 & 0.89 \\
$\begin{array}{l}\text { Self-completed ( } n=24) \\
\begin{array}{l}\text { Decrease in appetite } \\
\text { Researcher-completed } \\
(n=25)\end{array}\end{array}$ & 25 & 0 & & \\
$\begin{array}{l}\text { Self-completed }(n=23) \\
\text { Feeling different in yourself }\end{array}$ & 18 & 5 & -2.80 & 0.005 \\
$\begin{array}{l}\text { Researcher-completed } \\
(n=35)\end{array}$ & 35 & 0 & & \\
\begin{tabular}{l} 
Self-completed $(n=29)$ \\
\hline
\end{tabular} & 27 & 2 & -1.64 & 0.10 \\
\hline
\end{tabular}

(decrease in appetite). Missing data for the demographic and STAI items are shown in Table 3.

4 Process of administering the tool Duration of completion is shown in Table 4. This showed no significant difference between the groups $\left(X^{2}=2.23, \mathrm{p}=0.33\right)$. A greater proportion of participants in the researcher-completed group took $>15$ minutes. Help was provided to $46 / 93$ (49.5\%) of the self-complete group. The researchers reported that only 10/108 (9.3\%) of the researcher-completed group found the questions difficult. The researchers administering the tool did not report that any participants in the researcher-completed group were made anxious. The tool was completed in a separate room for $122(60.1 \%)$, in an open area for $53(26.4 \%)$, and whilst receiving treatment for eight (4.0\%). Data were missing for 18 (9.0\%).

5 Comparison of patient questionnaires against GP records

Data from 51 patients were compared against their GP records (30 from the researcher-completed group, and 21 from the self-completed group). Of these: 16 had breast cancer; six colorectal; six lung; four endometrial; three each for haematological, pancreatic, prostate, and renal/bladder; and two testicular. The 51 patients had 74 consultations available for comparison with the GP records. 28 patients had one consultation available, 13 had two, five had three, three had four, and two had five. The findings are shown in Figure 2. This shows that there was greater evidence of consultations being recorded in the GP records for participants who reported an exact date rather than for those who reported an estimated date. For exact dates there was evidence in the GP records of a consultation on the same date or within the proscribed time window for 32/37 (86\%) consultations. For estimated dates there was evidence for $23 / 37$ (62\%) consultations within the proscribed time window.

\section{Discussion}

\section{Summary of main findings}

We have developed the Cancer Symptom Interval Measure (C-SIM) for collecting patient-reported data relating

Table 3 Missing data regarding the demographic questions and STAI items

\begin{tabular}{|c|c|c|c|c|}
\hline & $\begin{array}{l}\text { Self-completed } \\
\qquad n=93\end{array}$ & $\begin{array}{c}\text { Researcher- } \\
\text { completed } \\
n=108\end{array}$ & z statistic & $\mathbf{P}$ \\
\hline \multicolumn{5}{|l|}{ Demographic questions } \\
\hline Employment & $3(3.2 \%)$ & $0(0.0 \%)$ & 1.88 & 0.06 \\
\hline Educational level & $5(5.4 \%)$ & $3(0.9 \%)$ & 0.94 & 0.35 \\
\hline Ethnicity & $2(2.2 \%)$ & $0(0.0 \%)$ & 1.53 & 0.13 \\
\hline Domestic status & $2(2.2 \%)$ & $1(0.9 \%)$ & 0.71 & 0.48 \\
\hline Smoking & $2(2.2 \%)$ & $0(0.0 \%)$ & 1.53 & 0.13 \\
\hline At risk of cancer (family history) & $2(2.2 \%)$ & $1(0.9 \%)$ & 0.71 & 0.48 \\
\hline \multicolumn{5}{|l|}{ 6-item STAI } \\
\hline STAl item 1 & $7(7.5 \%)$ & $0(0.0 \%)$ & 2.90 & 0.004 \\
\hline STAl item 2 & $14(15.1 \%)$ & $0(0.0 \%)$ & 4.18 & 0.000 \\
\hline STAl item 3 & $17(18.3 \%$ & $0(0.0 \%)$ & 4.64 & 0.000 \\
\hline STAl item 4 & $10(10.8 \%)$ & $0(0.0 \%)$ & 4.64 & 0.000 \\
\hline STAl item 5 & $13(14.0 \%)$ & $1(0.9 \%)$ & 3.63 & 0.0003 \\
\hline STAl item 6 & $14(15.1 \%)$ & $0(0.0 \%)$ & 4.18 & 0.000 \\
\hline
\end{tabular}


Table 4 How long did it take the patient to compete the questionnaire?

\begin{tabular}{lcccc}
\hline & $\begin{array}{c}\text { Self-completed } \\
\mathbf{n}=\mathbf{9 3}\end{array}$ & $\begin{array}{c}\text { Researcher- } \\
\text { completed } \\
\mathbf{n}=\mathbf{1 0 8}\end{array}$ & $\boldsymbol{X}^{\mathbf{2}}$ & $\mathbf{P}$ \\
\hline 5-10 minutes & $16(17.2 \%)$ & $11(10.2 \%)$ & 2.23 & 0.33 \\
11-15 minutes & $35(37.6 \%)$ & $43(39.8 \%)$ & & \\
$>15$ minutes & $38(40.9 \%)$ & $50(46.3 \%)$ & & \\
Missing & $4(4.3 \%)$ & $4(3.7 \%)$ & & \\
\hline
\end{tabular}

to appraisal intervals, help-seeking intervals, and diagnostic intervals in the cancer diagnostic pathway for 11 separate cancers. This tool is easy to use and quick to complete. It has evidence for acceptability, feasibility, face validity and criterion validity. The main findings from the RCT were that there were lower anxiety levels in the researcher-completed group. It is not known whether the higher level of anxiety in the self-completed group was a result of the cancer diagnosis, or the completion of the questionnaire. Data completeness was also greater for the researcher-completed group for some of the items.

\section{Discussion of the findings within the context of the literature}

There are very few reports of the development of measures to measure diagnostic times in cancer [14]. We are not aware of any other studies that specifically measure anxiety within this context. Hence it is difficult to know whether the relatively elevated anxiety in the self-complete group is specific to this tool. A recent review demonstrated that there is a small but significant difference in subjective outcomes reported either through selfcompleted or interviewer-completed modes [23]. So the validity of response is partly driven by that feature of data collection mode. Because of that it may be that some of the difference in anxiety found between forms of completion in this study could be attributable not only to the calming (or similar) effect of the researcher, but also due to associated response bias. For example, this could be that patients giving answers to researchers do not want to show their feelings whilst those selfcompleting forms are being more 'honest'-a form of social desirability bias. Unger-Saldana et al have recently reported the development and validation of a questionnaire to assess treatment delays in breast cancer [25]. Their researcher-administered tool was aimed at lower socioeconomic groups, and included many domains about the diagnostic process; hence is very different from our tool. Corner et al reported similar levels of reporting of the date of consultation between patients' recall and evidence from general practice records (Kendall's tau-b $=0.65$ ) [26].

\section{Strengths and weaknesses}

This was an adequately powered RCT with an overall completion rate of $75 \%$, and hence with a low risk of bias [27]. As anticipated, there was a higher degree of missing data in the anxiety domain from the selfcompleted group. We did not assess anxiety prior to administration of the tool, hence there is the possibility that the anxiety levels in the groups were different at baseline, Whilst we have assumed that the missing data were missing completely at random, these individuals did exhibit a greater degree of anxiety and there could be a theoretical association between anxiety and completion. The generalisability of the findings is limited with regard to ethnicity, as the sample was almost exclusively white.

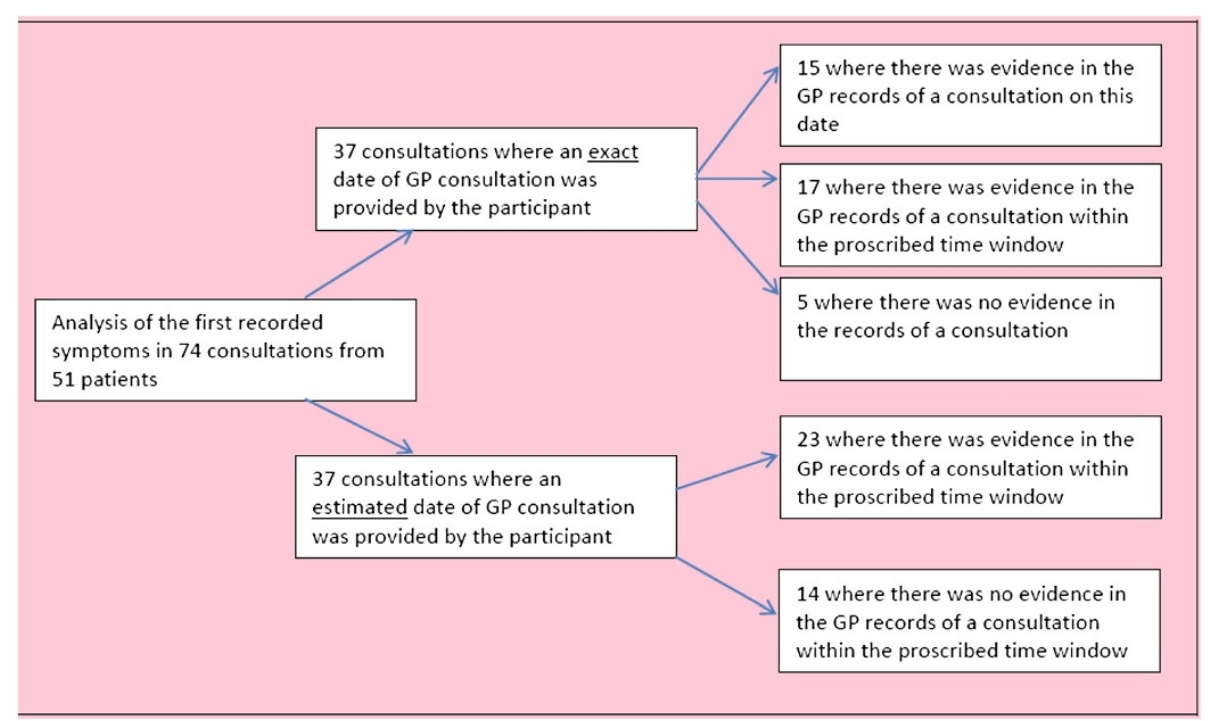

Figure 2 Validation of patient questionnaires against GP records. 


\section{Implications for policy, practice and research}

Given the lack of evidence of validation of tools in this area, we are happy for the Cancer Symptom Interval Measure (C-SIM) to be used in future descriptive and epidemiological studies of cancer diagnostic journeys, and to assess the functioning of current diagnostic service provision. Given our findings of less anxiety and more complete data collection in the researcher-completed group, we would recommend the researcher-administered approach where feasible. However, this clearly has resource implications, and limits its use in population-based studies. The agreement between patient questionnaires and GP records was moderate and whilst there was greater evidence of consultations being recorded in the GP records, for participants who reported an exact date rather than an estimated date, for many consultations the participants were unable to report an exact date, hence keeping both is probably necessary. Further work is needed to refine the tool in the light of the emergent findings, as some questions are easier to complete than others. Further research could also look at what may serve to reduce anxiety by researcher-administered forms. This could include having a professional and supportive presence when completing the form.

\section{Conclusion}

We have developed and tested a tool for collecting patient-reported data relating to appraisal intervals, help-seeking intervals, and diagnostic intervals in the cancer diagnostic pathway for 11 separate cancers, and provided evidence of its acceptability, feasibility and validity. This is a useful tool to use in descriptive and epidemiological studies of cancer diagnostic journeys, and causes less anxiety if administered by a researcher.

\section{Additional files}

Additional file 1: The colorectal tool.
Additional file 2: Cancer specific questions for all cancers.
Additional file 3: Protocol for calculating 'pseudo-exact' time
windows from estimated dates for the validation.
Additional file 4: Protocol for calculating 'pseudo-exact' dates from
estimated dates to calculate time intervals.

\section{Competing interests}

The authors declare that they have no competing interest in the conduct of this study.

\section{Authors' contributions}

$\mathrm{RDN}, \mathrm{DP}, \mathrm{KH}, \mathrm{MH}, \mathrm{MM}, \mathrm{CW}$, and $\mathrm{MR}$ designed the study and obtained the funding. $\mathrm{BC}$ conducted the randomisation. SN and DP conducted the fieldwork, with input from RDN, MH, NW, SG, MM and NS. BC, JT and KH undertook the analysis. RDN wrote the first draft of the paper, and all co-authors have approved the final draft of the manuscript. RDN will act as guarantor. All authors read and approved the final manuscript.

\section{Acknowledgements}

We would like to thank all of the participants in the trial and the patients, and patient groups, who helped us with the development of the tool. We would also like to thank the research staff from CRC Cymru / NISCHR (especially Angela Evans) for recruiting and administering the tool. SEWTU is funded by the National Institute for Social Care and Health Research. Our reporting is in line with the CONSORT statement. The C-SIM is free to use but only with permission from the lead author.

\section{Ethics, funding \& trial registration}

The trial was funded by a project grant from Cancer Research UK C8350/ A8205. Ethical approval was received from North Wales East Research Ethics Committee 07/NNo03/1. Registered trial number ISRCTN04475865.

\section{Author details}

${ }^{1}$ North Wales Centre for Primary Care Research, Bangor University, Gwenfro Unit 5, Wrexham Technology Park, Wrexham LL13 7YP, UK. ${ }^{2}$ School of Medicine, Cardiff University, Neuadd Meirionnydd, Heath Park, Cardiff CF14 4YS, UK. ${ }^{3}$ Betsi Cadwaladr University Health Board, Ysbyty Gwynedd, Penrhosgarnedd, Bangor, Gwynedd LL57 2PW, UK.

Received: 26 July 2013 Accepted: 20 December 2013

Published: 3 January 2014

\section{References}

1. Moller $\mathrm{H}$, Linklater KM, Robinson D: A visual summary of the EUROCARE-4 results: a UK perspective. Br J Cancer 2009, 101(suppl):110-114.

2. Abdel-Rahman M, Stockton D, Rachet D, Hakulinen T, Coleman MP: What if cancer survival in Britain were the same as in Europe: how many deaths are avoidable? Br J Cancer 2009, 101(suppl):115-124.

3. Richards MA: The national awareness and early diagnosis initiative in England: assembling the evidence. Br J Cancer 2009, 101(suppl):1-4.

4. Richards MA: The size of the prize for earlier cancer diagnosis of cancer in England. Br J Cancer 2009, 101(suppl):125-129.

5. Allgar VL, Neal RD: Delays in the diagnosis of six cancers: analysis of data from the national survey of NHS patients: cancer. Br J Cancer 2005, 92:1959-1970.

6. Hamilton W: Five misconceptions in cancer diagnosis. Br J Gen Pract 2009, 59:441-447.

7. Department of Health: The national survey of NHS patients-cancer. London: Department of Health; 2002.

8. Elliss-Brookes L, McPhail S, Ives A, Greenslade M, Shelton J, Hiom S, Richards M: Routes to diagnosis for cancer-determining the patient journey using multiple routine data sets. Br J Cancer 2012, 107:1220-1226.

9. Neal RD: Do diagnostic delays in cancer matter? Br J Cancer 2009, 101(suppl):9-12.

10. Tørring ML, Frydenberg M, Hansen RP, Olesen F, Hamilton W, Vedsted P: Time to diagnosis and mortality in colorectal cancer: a cohort study in primary care. Br J Cancer 2011, 104:934-940.

11. Andersen RS, Vedsted P, Olesen F, Bro F, Søndergaard J: Patient delay in cancer studies: a discussion of methods and measures. BMC Health Serv Res 2009, 9:189.

12. Scott SE, Walter FM, Webster A, Sutton S, Emery JD: The model of pathways to treatment: conceptualisation and integration with existing theory. Br J Health Psych 2013, 18:45-65.

13. Walter FM, Scott SE, Webster A, Emery JD: The Andersen model of total patient delay: a systematic review of its application in cancer diagnosis. J Health Serv Res \& Policy 2012, 17:110-118.

14. Weller D, Vedsted P, Rubin G, Walter F, Emery J, Scott S, Campbell C, Andersen RS, Hamilton W, Olesen F, Rose P, Nafees S, Van Rijswijk E, Muth C, Beyer M, Neal RD: The Aarhus statement: improving design and reporting of studies on early cancer diagnosis. Br J Cancer 2012, 106:1262-1267.

15. Neal RD, Pasterfield DM, Wilkinson C, Hood K, Makin M, Lawrence H: Determining patient and primary care delay in the diagnosis of cancer-lessons from a pilot study of patients referred for suspected cancer. BMC Fam Pract 2008, 9:9.

16. Fitzpatrick R, Davey C, Buxton MJ, Jones DR: Evaluating patient-based outcome measures for use in clinical trials. Health Technol Assess 1998, 2:14.

17. Streiner DL, Norman GR: Health measurement scales: a practical guide to their development and use. 4th edition. Oxford: Oxford University Press; 2008. 
18. Jobe JB: Cognitive psychology and self-reports: models and methods. Qual Life Res 2003, 12:219-227.

19. Willis GB: Cognitive Interviewing: a tool for improving questionnaire design. Thousand Oaks: Sage Publications; 2005.

20. Tourangeau R, Rips LJ, Rasinski K: The psychology of survey response. Cambridge: Cambridge University Press; 2000.

21. Smeeth $L$, Fletcher $A E$ : Improving the response rates to questionnaires. BMJ 2002, 324:1168-1169.

22. Edwards P, Roberts I, Clarke M, DiGuiseppi C, Pratap S, Wentz R, Kwan I: Increasing response rates to postal questionnaires: systematic review. BMJ 2002, 324:1183.

23. Hood K, Robling M, Ingledew D, Gillespie D, Greene G, Ivins R, Russell I, Sayers A, Shaw C, Williams J: Mode of data elicitation, acquisition and response to surveys: a systematic review. Health Technol Assess 2012, 16:27.

24. Marteau TM, Bekker $\mathrm{H}$ : The development of a six-item short-form of the state scale of the Spielberger state-trait anxiety inventory (STAI). Br J Clin Psychol 1992, 31:301-306.

25. Unger-Saldana K, Pelaez-Ballestas I, Infante-Castaneda C: Development and validation of a questionnaire to assess delay in treatment of breast cancer. BMC Cancer 2012, 12:626.

26. Corner J, Hopkinson J, Fitzsimmons D, Barclay S, Muers M: Is later diagnosis of lung cancer inevitable? Interview study of patients' recollections of symptoms before diagnosis. Thorax 2005, 60:314-319.

27. Higgins JPT, Green S: Cochrane handbook for systematic reviews of interventions. Cochrane Collaboration 2011, Version 5.1.0. Available from http://www.cochrane.org/training/cochrane-handbook.

doi:10.1186/1472-6963-14-3

Cite this article as: Neal et al:: Patient-reported measurement of time to diagnosis in cancer: development of the Cancer Symptom Interval Measure (C-SIM) and randomised controlled trial of method of delivery. BMC Health Services Research 2014 14:3.

\section{Submit your next manuscript to BioMed Central and take full advantage of:}

- Convenient online submission

- Thorough peer review

- No space constraints or color figure charges

- Immediate publication on acceptance

- Inclusion in PubMed, CAS, Scopus and Google Scholar

- Research which is freely available for redistribution 